\title{
The way we view cellular (glyco)sphingolipids
}

\author{
Sandra Hoetzl, Hein Sprong and Gerrit van Meer \\ Membrane Enzymology, Bijvoet Center and Institute of Biomembranes, Utrecht University, Urecht, The Netherlands
}

\begin{abstract}
Mammalian cells synthesize ceramide in the endoplasmic reticulum (ER) and convert this to sphingomyelin and complex glycosphingolipids on the inner, non-cytosolic surface of Golgi cisternae. From there, these lipids travel towards the outer, non-cytosolic surface of the plasma membrane and all membranes of the endocytic system, where they are eventually degraded. At the basis of the selective, anterograde traffic out of the Golgi lies the propensity of the sphingolipids to selfaggregate with cholesterol into microdomains termed 'lipid rafts'. At the plasma membrane surface these rafts are thought to function as the scaffold for various types of (glyco) signaling domains of different protein and lipid composition that can
\end{abstract}

co-exist on one and the same cell. In the past decade, various unexpected findings on the sites where sphingolipid-mediated events occur have thrown a new light on the localization and transport mechanisms of sphingolipids. These findings are largely based on biochemical experiments. Further progress in the field is hampered by a lack of morphological techniques to localize lipids with nanometer resolution. In the present paper, we critically evaluate the published data and discuss techniques and potential improvements.

Keywords: glycolipids, immuno electron microscopy, immunofluorescence microscopy, lipid topology, subcellular localization.

J. Neurochem. (2007) 103 (Suppl. 1), 3-13
Sphingolipids form a highly diverse class of lipids, characterized by a lipid backbone that consists of a long-chain sphingoid base that is mostly amide-linked to a fatty acid: ceramide. The polar headgroup of the most abundant sphingolipid in mammalian cells, sphingomyelin (SM), is phosphocholine. It is transferred from the cell's most abundant glycerophospholipid phosphatidylcholine (PC) onto ceramide by sphingomyelin synthase on the lumenal side of the Golgi membrane, giving rise to SM plus diacylglycerol. Both ceramide and diacylglycerol fulfill dual functions as metabolic precursors and as secondary messengers. The glycosphingolipids contain polar headgroups that contain one or more carbohydrates. The basic glycosphingolipid in every cell is glucosylceramide (GlcCer; Table 1), to which carbohydrates are added in a stepwise fashion on the lumenal surface of the Golgi. This yields glycosphingolipids containing one up to ten or more carbohydrates, which are often organized as branched chains. Glycosphingolipids containing sialic acids are termed gangliosides. Independently, a number of cell types synthesize galactosylceramide (GalCer), part of which is modified in the trans Golgi lumen to GalCer sulfate (sulfatide). These latter lipids are relevant for the proper function and stability of the myelin sheath, as evidenced by the tremor, late-onset paralysis and premature death of knockout mice for the ceramide GalCer synthase. The GlcCer-based glycosphingolipids are crucial for mammalian development.
Once again, this has become clear from studies on knock-out mice. Mice with null-alleles for the GlcCer synthase, causing a lack of all complex glycosphingolipids (Yamashita et al. 1999), die as embryos. Mice lacking more distal transferases display milder defects, which however, when translated to humans, would still be recognized as major inherited diseases (Proia 2003). For example, whereas mice lacking the GM3 synthase displayed enhanced insulin sensitivity (Yamashita et al. 2003), humans with this defect display infantile-onset symptomatic epilepsy syndrome (Simpson et al. 2004). While SM generally makes up some $15-20 \%$ of the lipids in plasma membranes, the levels of glycosphingolipids are generally much lower. However, particularly high levels of glycosphingolipids have been reported for apical membranes of epithelial cells (Simons and van Meer 1988), for myelin (Bosio et al. 1996; Coetzee et al. 1996) and neurons (Vanier 1999).

The molecular basis for the need for glycosphingolipids was originally supposed to reside in the tremendous variability in three-dimensional structure, that can be

Address correspondence and reprint requests to Gerrit van Meer, Membrane Enzymology, Bijvoet Center and Institute of Biomembranes, Utrecht University, Padualaan 8, 3584 CH Utrecht, The Netherlands. E-mail: g.vanmeer@uu.nl

Abbreviations used: ER, endoplasmic reticulum; GlcCer, glucosylceramide; LacCer, lactosylceramide. 
Table 1 Glycosphingolipid designation according to the recommendations of the IUPAC-IUB Joint Commission on Biochemical Nomenclature ${ }^{a}$, using the Svennerholm abbreviations for gangliosides ${ }^{b}$

\begin{tabular}{|c|c|c|c|}
\hline Abbreviation & Name & Structure & Synonyms \\
\hline GlcSph & Glucosylsphingosine & Glc $\beta 1-S p h$ & Glucopsychosine \\
\hline GalSph & Galactosylsphingosine & Gal $\beta 1-S p h$ & (galacto)Psychosine \\
\hline GlcCer & Glucosylceramide & Glc $\beta 1-$ Cer & Glucocerebroside \\
\hline GalCer & Galactosylceramide & Gal $\beta 1-$ Cer & (galacto) Cerebroside \\
\hline LacCer & Lactosylceramide & Gal $\beta 4$ Glc $\beta 1-$ Cer & \\
\hline SGalCer & Sulfogalactosylceramide & $\mathrm{HSO}_{3}-3 \mathrm{Gal} \beta 1-\mathrm{Cer}$ & Sulfatide, SM4 \\
\hline SLacCer & Sulfolactosylceramide & $\mathrm{HSO}_{3}-3 \mathrm{Gal} \beta 4 \mathrm{Glc} \beta 1-\mathrm{Cer}$ & SM3 \\
\hline Gb3 & Globotriaosylceramide & Gal $\alpha 4$ Gal $\beta 4$ Glc $\beta 1-$ Cer & $\mathrm{Gb}_{3} \mathrm{Cer}$ \\
\hline \multicolumn{4}{|l|}{ Gangliosides } \\
\hline GM1a & $\|^{3} \mathrm{NeuAc}-\mathrm{Gg}_{4} \mathrm{Cer}$ & Gal $\beta 3$ GalNAc $\beta 4(N e u 5 A c \alpha 3)-G a l \beta 4$ Glc $\beta 1$ Cer & GM1 \\
\hline GM1b & $\mathrm{IV}^{3} \mathrm{NeuAc}-\mathrm{Gg}_{4} \mathrm{Cer}$ & Neu5Ac $\alpha 3 G a l \beta 3 G a l N A c \beta 4-G a l \beta 4 G \mid c \beta 1$ Cer & $\mathrm{G}_{\mathrm{M} 1 \mathrm{~b}}$ \\
\hline GM3 & $\| I^{3} \mathrm{NeuAc}-$ LacCer & Neu5Ac $\alpha 3$ Gal $\beta 4$ Glc $\beta 1$ Cer & $\mathrm{G}_{\mathrm{M} 3}$ \\
\hline GD3 & $\| I^{3} \mathrm{NeuAc}_{2}-$ LacCer & Neu5Ac $\alpha 8$ Neu5Ac $\alpha 3-G a l \beta 4 G \mid c \beta 1$ Cer & $\mathrm{G}_{\mathrm{D} 3}$ \\
\hline GM4 & $I^{3} \mathrm{NeuAc}-\mathrm{GalCer}$ & Neu5Ac $\alpha 3 G a \mid \beta 1 C e r$ & $\mathrm{G}_{\mathrm{M} 4}$ \\
\hline
\end{tabular}

${ }^{\mathrm{a}}$ Chester (1998), ${ }^{\mathrm{b}}$ Svennerholm (1963).

generated by combining various carbohydrates in different combinations and orders and with different glycosidic bonds. Already early on, this variability suggested that the oligosaccharides on glycoproteins and glycosphingolipids might serve to mediate highly specific interactions between cells, cells and matrix and between cells and soluble signaling molecules (see Hakomori 2002). The high structural specificity is illustrated by the fact that many natural high-affinity antibodies have turned out to be directed against specific glycosphingolipids instead of proteins. An alternative role for sphingolipids was suggested by the lipid raft hypothesis in the late 1980s. The original hypothesis stated that glycosphingolipids in the lumenal leaflet of the membrane of the trans Golgi network self-aggregate and that epithelial cells selectively include these aggregates into budding transport vesicles destined for the apical plasma membrane domain of epithelial cells, sorting glycolipids with the apical proteins (van Meer et al. 1987). With the inclusion of SM and cholesterol, the hypothesis also nicely explains how cells enrich sphingolipids and cholesterol in the anterograde secretory pathway and thereby keep the endoplasmic reticulum (ER) concentrations of these lipids low (Fig. 1). The apical sorting of glycosphingolipids would then be superimposed on the general sorting of all sphingolipids and cholesterol towards the plasma membrane (van Meer 1989). Because the complex sphingolipids reside on the non-cytosolic surface they can reach other organelles by vesicular transfer only. The absence of a vesicular transport connection to mitochondria and peroxisomes then explains the general lack of SM and glycosphingolipids from these membranes as assessed biochemically (van Meer 1989). Because cholesterol readily flips across membranes and has a relatively high rate of monomeric transfer between membranes, its cellular distribution is determined essentially by

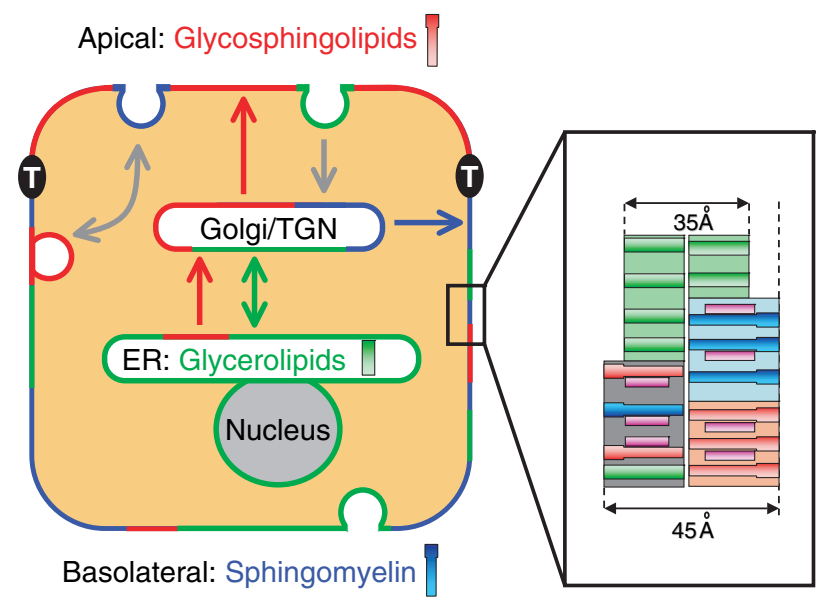

Fig. 1 Lipid sorting in intestinal epithelial cells (Simons and van Meer 1988; van Meer 1989). Glycosphingolipids (red) and cholesterol are enriched on the apical surface, whereas SM (blue) with cholesterol is preferentially transported to the basolateral surface. All three lipids are sorted in the anterograde direction, while unsaturated glycerophospholipids (green) are enriched in the retrograde pathway to the ER. The sorting occurs in the non-cytosolic leaflet of the various membranes, because the tight junctions $(\mathrm{T})$ act as a barrier to lipid diffusion in the outer bilayer leaflet only. However, some sphingolipids are presumably present in the cytosolic leaflet. For the differences in membrane thickness (see Sprong et al. 2001).

its high affinity for sphingolipids (Wattenberg and Silbert 1983). (Glyco)sphingolipid-based rafts have received most attention for their roles in the signaling by immune receptors (Gomez-Mouton et al. 2001; Pierce 2002; Holowka et al. 2005; Barbat et al. 2007), in glycosignaling platforms on the cell surface (Hakomori 2002), and in other signal transduction events (see Mayor et al. 2006). 
In the past decade, various unexpected genetic and biochemical findings have opened new vistas on the organization of sphingolipids in the mammalian cell bringing up as many new sets of questions on the topology of the respective sphingolipids. (i) The simple glycosphingolipid glucosylceramide (GlcCer) is not synthesized in the lumenal leaflet of the Golgi membrane, but on the cytosolic surface (Ichikawa et al. 1996). Across which membrane does GlcCer translocate on its way into the Golgi lumen where it is required for the synthesis of higher glycosphingolipids? (ii) In contrast, the ceramide galactosyltransferase synthesizes galactosylceramide (GalCer) on the lumenal surface of the ER (Schulte and Stoffel 1993), where like all other lipids tested it has free access to the cytosolic surface by an energyindependent flip-flop process (Fig. 2). Do both GlcCer and GalCer redistribute to all other cytosolic surfaces via, for example, the cytosolic glycolipid transfer protein GLTP? (iii) Biochemically, complex glycosphingolipids and SM are found to some extent in the ER and even in the nuclear matrix (Albi and Viola Magni 2004; Hunt and Postle 2006). Are they really there and do they, as a consequence, have access to the cytosolic surface of the ER and that of other organelles as well? Recently, a non-lysosomal GlcCer degrading enzyme has been found in the ER (Boot et al. 2007). (iv) While mitochondria contain exceedingly low concentrations of (glyco)sphingolipids, in apoptotic cells SM and the ganglioside GD3 do seem to reach the mitochondria

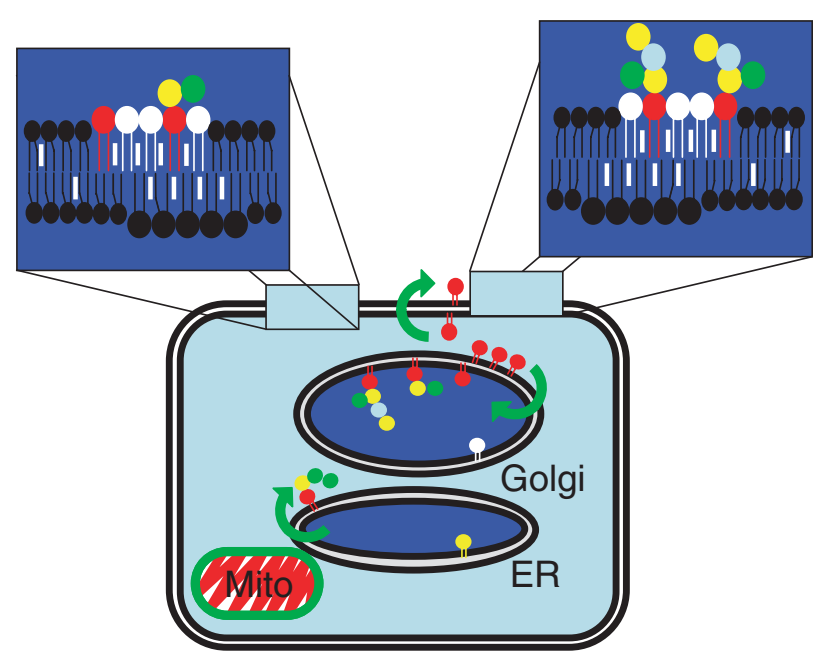

Fig. 2 Glucosylceramide (GlcCer; red) is synthesized on the cytosolic surface of the Golgi but is flipped to the lumenal surface of the Golgi, where it is converted to complex glycosphingolipids by the sequential addition of galactose (yellow) and sialic acid (green), to yield GM3. GM1 is synthesized by the further addition of $\mathrm{N}$-acetylgalactosamine (light blue) and another galactose (Table 1). GM3 and GM1 occupy different domains on the surface of polarizing T cells, probably with SM (white). Some GD3 makes it back to the ER, where it can presumably flip freely across the membrane and now has access to, for example, mitochondria via contact sites. and to cause mitochondrial rupture and dysfunction (Rippo et al. 2000; Birbes et al. 2001; Garcia-Ruiz et al. 2002). Where exactly are these lipids located and how do these processes work? (v) The biosynthesis of SM and part of the GlcCer depends on transport of newly synthesized ceramide from the ER to the trans Golgi by the ceramide transfer protein CERT (Hanada et al. 2003). Does CERT act as a soluble carrier or as a contact-site protein, and where exactly does it deliver the ceramide? (vi) Finally, evidence has been presented that different types of microdomains can co-exist on the cell surface and that these domains have different (sphingo)lipid compositions (Gomez-Mouton et al. 2001; Brügger et al. 2004). How are these domains structured and do such domains exist along the vesicular transport pathways as well? These examples clearly illustrate the need for data on the location of (glyco)sphingolipids, if possible with $\mathrm{nm}$ resolution.

\section{Localization of (glyco)sphingolipids; the methods}

So, what do we want to know about where the sphingolipid is located in the in vivo situation? In increasing order of resolution: (i) In which tissue or part of the tissue? (ii) In which cells? (iii) In which membranes in the cells? (iv) On which side of the membrane? (v) And, finally, at which lateral location within that membrane leaflet? At that point, we have reached the realm of chemistry and physics, where we can ask with which other membrane components our lipid interacts. Because natural lipids are not visible in the microscope (except for the very few that are autofluorescent), two general approaches and the combination of them have been followed to visualize them. (i) Lipids have been labeled by reagents that can be discriminated microscopically, mostly proteins that bind to a certain lipid, like antibodies, followed by fluorescent or gold-labeled secondary antibodies; (ii) analogs of lipids have been inserted into cells that are visible by themselves, because they are fluorescent, or can be visualized by the previous approach.

\section{Labeling of endogenous lipids}

Because of the remarkable antigenicity of glycosphingolipids, many anti-glycosphingolipid antibodies have been described. After binding to their target, they can be visualized by the traditional methods of immunofluorescence and immunoelectron microscopy. In comparison to the well-established immunolocalization of proteins, the localization of lipids is hampered by a number of special technical problems connected with their small size and hydrophobic nature. As a general principle, the burden is on the investigator to make sure that the interpretation adequately recognizes these problems. Only meaningful data can lay the solid foundation for the field that is needed before we can start to discuss the effects of lipids on the cellular organization, trafficking and signaling of proteins at the molecular level. 


\section{Access without loss or redistribution}

The first and overriding condition that should be fulfilled by any method is that the method should not affect the feature that is to be studied, which in this case is the localization of the sphingolipid in the living cell. So the method should not change the level of the lipid or redistribute the lipid. The concentration of the lipid may change due to metabolism during the labeling procedure. This is especially realistic for signaling lipids which have a very high turnover. In addition, some enzymes are particularly resistant to fixatives. In these cases there are still various possibilities to record the in vivo situation: (i) to label in vivo, and study the label in vivo under a fluorescence microscope, (ii) to label in vivo, and fix the label instead of the lipid, and (iii) to freeze the situation by rapid freezing after which the lipids must be fixed in the frozen state.

A second problem is accessibility. Only glycosphingolipids on the cell surface can be labeled directly by exogenous antibodies. When one tries to approach intracellular lipids, problems arise that do generally not exist for protein targets. For protein localization, proteins are first fixed by aldehydes after which they are made accessible to the antibodies by opening the membranes. Because lipids are generally not fixed by aldehydes (and if they are, they mostly lose their antigenicity) special care must be taken to ensure that the opening method does not displace (or remove) the lipids. First of all, membranes have been opened by detergents or organic solvents like cold methanol or acetone. Such methods generally result in dissolution and release (Maneta-Peyret et al. 1999) and redistribution of lipids. This point has been convincingly made even at the level of tissues. The incubation with low concentrations of Triton X-100 resulted in the redistribution of glycosphingolipids between brain tissue samples that were co-processed in the same solution (Heffer-Lauc et al. 2005). Subcellular labeling patterns of lipids observed after such treatments must be verified by independent techniques. It should be realized that even a fixation procedure using aldehydes by itself can make membranes leaky to the labeling agent (Sugii et al. 2003). Such a change in the physical state of the membrane may well induce redistribution of lipids across, or in the plane of, the membrane or even between organelles.

A detergent-independent, general method to make proteins accessible for localization is the preparation of sections from frozen samples, frozen sections, which is followed by antibody labeling on the thawed sections. It is clear from lipid physics that cross-sectioned, and thereby opened, membranes have an inherent strong tendency to close. The lipids on the edge of the cross-sectioned lipid bilayer are inevitably unstable (Fig. 3), resulting in lipid redistribution across membranes and high background labeling over the section (van Genderen et al. 1991). A dramatic improvement in organelle-specific labeling on sections was observed after freeze-substitution: freezing the cells, followed by embedding in a polymer that is subsequently cross-linked by UV, all below $0^{\circ} \mathrm{C}$. The complex glycosphingolipid Forssman antigen was found to reside in specific cellular membranes and to be absent from mitochondria and peroxisomes (van Genderen et al. 1991). First of all, this confirms that freezesubstitution does not redistribute the lipid over organelles, second, the results suggest that it is technically possible to address the question whether glycosphingolipids are present in mitochondria under specific conditions. The labeling density was high in the plasma membrane and in endosomes, threefold lower in the Golgi and 10-fold lower in the nuclear membrane. This fits the expectations from biochemical analyses, which suggested that this immunolocalization technique can be used to quantitate the surface density of the lipid in the various membranes. A later study using freeze-substitution (Parton 1994) even supplied sufficient resolution to conclude that the glycosphingolipid GM1 was not distributed uniformly over the plasma membrane but (a)

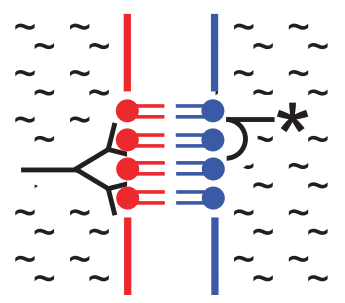

(b)

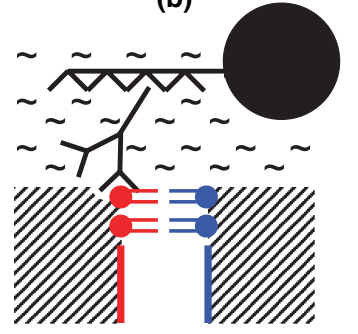

(c)

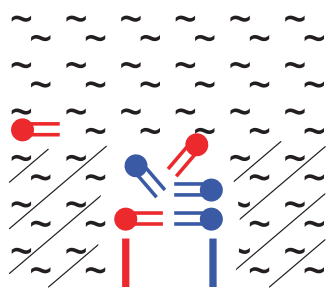

Fig. 3 The sidedness of lipid labeling. (a) Membrane impermeable probes can be added selectively from one side of the lipid bilayer. Examples are the exogenous addition of antibodies or toxins to the outer surface of intact cells (red; e.g., (Parton 1994), or the expression of cytosolic fluorescent $\mathrm{PH}$ domains to label phosphoinositides on cytosolic surfaces (blue; (Downes et al. 2005). Besides studying them by fluorescence, these labels can be fixed and processed for electron microscopy by a variety of techniques (thin frozen sections, plasma membrane sheets, fracture-label), whereby the label is visualized by pre- or post-embedding with, e.g., protein A-gold. (b) In freeze-substitution the samples are frozen, and the ice replaced by a polymer below $0^{\circ} \mathrm{C}$. Thin sections are cut, and a primary reagent (antibody) followed by a secondary reagent (protein A- $5 \mathrm{~nm}$ gold) labels lipids at the surface of the section. The gold does not reflect the sidedness of the lipid antigen. (c) In conventional thin frozen sections, the sample is thawed and labeled. As illustrated lipids at the interface will redistribute and some will spread. Labeling as in (b). While antibodies can penetrate the section, the gold label will not. 
was concentrated approximately fourfold in non-coated invaginations, identified as caveolae. This was the first morphological evidence for the occurrence of glycosphingolipid-enriched domains in a mammalian plasma membrane. (Vielhaber et al. 2001b) successfully demonstrated a concentration of GlcCer in lamellar bodies of the skin. Excellent preservation of the galactosphingolipids GalCer and S-GalCer to myelin sheets in rat brain was reported as well (Kirschning et al. 1998). Still, there are many variables in the fixation and embedding steps of the method that must be optimized to minimize lipid loss (Maneta-Peyret et al. 1999), and redistribution.

One other method to gain access to the cytosolic surface of the plasma membrane is the preparation of plasma membrane sheets. Labeling such sheets with gold-labeled antibodies, followed by imaging and pattern analysis allowed an assessment of the clustered appearance of lipid-anchored kinases on the cytosolic surface (Plowman et al. 2005), and an assessment of the lateral distribution of phosphoinositides and phosphatidylserine, lipids restricted to the cytosolic surface, should not be problematic: An analysis of the distribution of prelabeled GM1 (in the outer leaflet) showed a difference between the random distribution in the control and the clustering occurring after cross-linking (Wilson et al. 2004).

A final problem of accessibility is shielding of the lipid by either proteins or by other lipids. This particularly applies to lipids that are deeply embedded in the lipid bilayer and expose only a small headgroup. A clear example is the observation that the cholesterol-binding agent filipin does not bind to cholesterol in coated vesicles until after removal of the clathrin coat (McGookey et al. 1983; Steer et al. 1984). This predicts that agents proposed to label lipids like ceramide (Vielhaber et al. 2001a) may not label their target with similar efficiencies in different membranes or different membrane domains. The labeling of SM by the SM-binding toxin lysenin, or probes derived thereof, depended on the lipid environment. The presence of glycolipids inhibited binding (Ishitsuka et al. 2005). Such probes can therefore not be used for the quantitative assessment of the distribution of a lipid over various locations, because the absence of labeling does necessarily mean that the lipid target is not present.

\section{Labeling without redistribution}

A subsequent set of problems with the microscopic localization of glycosphingolipids is connected to the nature of the labeling reagents. First of all, the agent should be specific. A standard test for the specificity of the label for a certain glycosphingolipid is a binding assay on TLC plates, but it is impossible to test all lipids for cross reactivity. Moreover, a lipid is presented differently on a TLC plate and in a biomembrane: the possibility exists that a protein specific in a TLC assay cross-recognizes other epitopes in the cellular context. The ideal test to exclude such cross-reactivity is to study the same cells but lacking this specific glycosphingo- lipid (van Genderen et al. 1991). This is now facilitated by the availability of a cell line without glycosphingolipids (Ichikawa et al. 1996). Second, the agent should have a high affinity for the lipid in order to increase the labeling density. The affinity increases when the labeling reagent is multivalent, which takes us to the next problem: because lipids are not fixed by routine fixation protocols, they can be redistributed by multivalent ligands abolishing their original distribution pattern. This is especially true when these primary ligands are subsequently labeled by secondary ligands that are multivalent by themselves. Labeling of the complex glycosphingolipids globoside and Forssman antigen by an antibody, or labeling of GM1 by cholera toxin, followed by tetra- or pentavalent protein A or by a second antibody resulted in clustering (Tillack et al. 1983; Spiegel et al. 1984; Butor et al. 1991; Fujimoto 1996). The artificial clustering by the second protein label could be prevented by fixation of the first label before addition of the second (Butor et al. 1991; Fujimoto 1996). A disturbance of the lipid distribution may also occur as a consequence of the fact that the reagent changes an essential physical property of the lipid. The label is generally big as compared to the target lipid and may change its diffusion co-efficient. Especially, labels that insert into the membrane like (agents based on) membrane penetrating toxins, e.g., lysenin for SM and perfringolysin for cholesterol, are likely to interfere with those lipid-lipid interactions that determine their phase behavior, and thereby affect their lateral distribution. Whereas the cellular dynamics of phosphoinositides have been studied extensively using the (green fluorescent protein-coupled) truncated binding domains (PH-, PX-, ENTH-, and FYVEdomains) of proteins that bind specifically to distinct phosphoinositides (Downes et al. 2005), remarkably few probes (antibodies/toxins) are available that bind one (glyco)sphingolipid molecule with sufficient affinity to study singular (glyco)sphingolipids.

Finally, the resolution of the labeling is limited by the dimensions of the labeling reagent: whereas the crosssectional area of a typical lipid is $0.6 \mathrm{~nm}^{2}$, the diameter of the gold particles is $5-10 \mathrm{~nm}$ or more, and the linker between the antigenic lipid and the gold particle again has a length of $>10 \mathrm{~nm}$ (Fig. 3). It is therefore of paramount importance to devise monovalent high-affinity reagents of a limited size. Still, it is clear that the size of the electron microscopic reagents by themselves makes it impossible to define the sidedness of an epitope on membranes by the post-embedding of thin sections (Fig. 3). Protocols must be used where the accessibility to the labeling reagent is limited to one side of the membrane only. This can be achieved by labeling intact membranes (or cells) prior to freezing, embedding or cutting. Alternative approaches that have been applied to lipids are the immunolabeling of membrane sheets (Wilson et al. 2004), and the fracture-label technique, which is immunolabeling of membrane halves produced by freezefracture (Barbosa and Pinto da Silva 1983). 


\section{Fluorescent lipid analogs}

Thirty years ago Simoni and colleagues demonstrated how parinaric acid, a family of unnatural fluorescent $\mathrm{C} 18$ fatty acids with 4 conjugated cis- and trans-double bonds can be used to study physical properties of fatty acids and phospholipids in vitro and in vivo (Sklar et al. 1975; Rintoul and Simoni 1977). Since then, a variety of fluorescent lipophilic dyes have been applied that unlike parinaric acid are visible by fluorescence microscopy. They have been used in numerous cases to study physical properties of cellular membranes and to unravel transport pathways of cellular lipids, for example the barrier properties of epithelial tight junctions (Dragsten et al. 1981; van Meer and Simons 1986). However, an important breakthrough in this field was the broad application by (Pagano et al. 1981) of visible fluorescent lipids that sufficiently resembled the natural mammalian lipids in structure to be recognized by the cellular metabolic pathways. Because these analogs carried a short fluorescent and relatively polar fatty acid (C6-NBD), they were rather well soluble in water, which property enabled the investigators to efficiently deliver them to, or deplete them from, the cell surface. Over the years, these probes provided a wealth of topological data (Pagano and Sleight 1985) and laid the basis for the lipid raft hypothesis (van Meer et al. 1987).

By the time that it became clear that the C6-NBD lipids did not faithfully reflect the phase properties of especially the raft forming sphingolipids (Wang and Silvius 2000), a new fluorescent fatty acid analog was introduced, C5-Bodipy, that is more hydrophobic and has the attractive possibility that its fluorescence is concentration-dependent (Pagano et al. 1991). Its use has provided information on glycolipid concentration into microdomains in the endocytic pathway (Sharma et al. 2003), to which picture also other fluorescent probes had contributed [reviewed in Mukherjee and Maxfield (2000); Barbat et al. (2007)]. Still, the phase properties of even the Bodipy-sphingolipids are different from those of their natural analogs (Wang and Silvius 2000), and the question remains how their localization in cells relates to that of the natural sphingolipids.

A more recent breakthrough in the application of fluorescent analogs of the cellular lipids takes us back full circle to the use of parinaric acids. In the intervening 30 years new delivery technologies and improved microscopy have potentiated the use for cell biology of pyrene fatty acids, as being more stable analogs than the parinaric acids while maintaining close-to-natural properties (Somerharju 2002). However, the parinaric acids have now been rendered suitable for light microscopy by the chemical addition of yet another conjugated double bond, which brings their excitation and emission wavelengths in the visible part of the spectrum. These fluorescent fatty acids are the first ones to be efficiently incorporated into cellular sphingolipids, illustrating their closest possible resemblance to the natural fatty acids and offering optimal possibilities to study the location and behavior of natural sphingolipids (Kuerschner et al. 2005). Still, there are numerous practical limitations to their broad application. The cunning combinations of biochemical, biophysical, and microscopic experiments devised over the years by numerous investigators for the application of other fluorescent lipid analogs should help to surmount these limitations and develop their utmost potential for the characterization of the topology of glycosphingolipids.

\section{Glycosphingolipid topology in mammalian cells}

Glycosphingolipids and SM have been found to be exposed on the surface of the cell by a number of biochemical approaches including enzymatic degradation, enzymatic and non-enzymatic oxidation, and the binding of antibodies and toxins. These data have led to the general impression that 60 $70 \%$ of the cellular SM and a large fraction of all glycosphingolipids are exposed on the cell surface, i.e., in the outer, non-cytosolic leaflet of the plasma membrane (see van Meer and Holthuis 2000; Sillence et al. 2000). This fits the overall idea that glycosphingolipids and SM are synthesized in the lumenal leaflet of the Golgi membrane and are transported via the inner leaflet of membrane vesicles (Fig. 2). As predicted from this idea these lipids follow the endocytotic recycling pathways and are also present on the lumenal side of membranes of endosomes and lysosomes (van Genderen et al. 1991), where they are finally degraded (Kolter and Sandhoff 2005).

\section{Diffusion barrier-based lipid macrodomains}

It has been realized for a long time that lipids on the surface of some cells display a polarized distribution. This is particularly true for epithelial cells from the intestine and the kidney where glycolipids or SM were found to be at least fourfold enriched in the apical domain of the continuous plasma membrane that surrounds those cells (see Simons and van Meer 1988; Holthuis et al. 2001). This situation is maintained by the proteinaceous tight junctions, which form a barrier to lipid diffusion in the outer leaflet of the plasma membrane. Thus, the differences in lipid composition reside in the outer leaflet and the glycosphingolipids and SM are enriched in the outer leaflet of the apical membrane [discussed in Simons and van Meer (1988)]. The other striking example of stable lipid macrodomains is found on the surface of sperm cells. In this case, the evidence points at a proteinaceous diffusion barrier between the acrosomal and post-acrosomal domain (James et al. 2004; Selvaraj et al. 2006).

\section{Microdomains based on lipid-lipid interactions}

Based on the enrichment of glycosphingolipids on the outside of the apical membrane and the preferential apical transport of various fluorescent glycosphingolipids as compared to sphingomyelin analogs in kidney and intestinal 
epithelial cells, it was proposed that these epithelial cells sort their lipids by the segregation of glycosphingolipids (and cholesterol) away from SM and phosphatidylcholine (plus cholesterol) in the lumenal leaflet of the trans Golgi network (van Meer et al. 1987; van 't Hof et al. 1992). It was then suggested that such lipid-based domains might exist at the cell surface where they could be involved in signal transduction (Lisanti et al. 1994). This original suggestion concerned stable structures, the caveolae, for which it was shown at that time that they were enriched in the glycosphingolipid GM1 (Parton 1994) by the application of cholera toxin on freeze-substituted samples, which was confirmed by photo-crosslinking (Fra et al. 1995). Stable glycosphingolipid-enriched domains may also exist in various types of glycosynapses (Hakomori 2002), e.g., noncaveolar glycosphingolipid signaling domains enriched in GM3 (Iwabuchi et al. 1998), and point contacts between neuronal growth cones and extracellular matrix (Negreiros et al. 2003). However, studies using a variety of biophysical techniques have suggested that microdomains of specific lipid compositions exist but that they are generally small and transient (Pike 2006), unless they are stabilized by an increase in order. Large scale lipid segregation may be induced by a change in curvature (Roux et al. 2005), or by changes occurring in membranes during signal transduction, like the generation of ceramide by a sphingomyelinase (Bollinger et al. 2005). Other factors causing lipid raft coalescence into stable platforms are the relatively subtle changes in lipid raft partitioning of multichain immune recognition receptors following signal initiation by ligandmediated receptor crosslinking (Pierce 2002; Holowka et al. 2005). In contrast, a different role for lipid rafts, namely as carriers for signaling molecules, has been suggested during the formation of the immunological synapse between antigen-presenting cells and T cells (Saito and Yokosuka 2006).

Sorice et al. (1997) properly post-fixed anti-GM3 antibodies before addition of the secondary antibodies to prevent redistribution of the primary antibodies upon cross-linking by the secondary IgM, and observed, by fluorescence and electron microscopy, large, $300 \mathrm{~nm}$ clusters of GM3 molecules that covered close to $50 \%$ of the surface of $\mathrm{T}$ cells. When (Spiegel et al. 1984) clustered GM1 into a cap on the lymphocyte surface via cholera toxin, anti-toxin antibodies and protein A, exogenously added (fluorescent) GM3 co-capped with GM1 suggesting that the two gangliosides resided in the same plasma membrane domains. However, Gomez-Mouton et al. (2001) reported that during T cell polarization GM1 and GM3 segregated into specific lipid rafts at the uropod and at the leading edge, respectively (Fig. 2). In polarizing MCF-7 adenocarcinoma cells GM1 moved to the leading edge (Manes et al. 1999), which probably also represents the uropod in these fibroblast-like cells (Gomez-Mouton et al. 2001). Finally, partial co-localization of GM1- and GM3- enriched domains was reported by Barbat et al. (2007) after the induction of CD4 signaling in the absence of $\mathrm{T}$ cell receptor engagement. However, electron microscopy and proper sample preparation protocols will be required to resolve whether there was real co-localization under these conditions. The same applies to the observation that GM1 and GD3 did not co-localize on cerebellar neurons (Vyas et al. 2001). Kiyokawa et al. (2005) concluded that GM1-rich membrane domains are spatially distinct from SM-rich domains on Jurkat $\mathrm{T}$ cells. However, because the SM-specific probe used, lysenin, does not bind to SM in the presence of glycosphingolipids (Ishitsuka et al. 2005), the possibility exists that (a high concentration of) SM was present in the GM1 rafts.

Evidence for the co-existence of different types of lipid rafts has also been reported for surface of neuronal cells. Two typical raft marker proteins, the glycosylphosphatidylinositol-anchored prion and Thy-1 proteins, occupied different domains. While prion protein occurred at high density in domains located primarily at the cell body, Thy-1 was clustered in separate domains mainly on neurites covering half of their surface (Madore et al. 1999). Affinity-purification of the two types of rafts from detergent-resistant membranes showed that prion protein rafts contained fivefold higher levels of glucosylceramide than Thy-1 rafts (Brügger et al. 2004).

\section{Intracellular sphingolipids}

In line with its synthesis in the lumen of the Golgi, the complex glycosphingolipid Forssman antigen was found in the Golgi, the plasma membrane and endosomal membranes, but not in mitochondria and peroxisomes (van Genderen et al. 1991). Obviously, high concentrations of glycosphingolipids can accumulate in endocytotic organelles in cells from patients suffering from a variety of storage diseases (Futerman and van Meer 2004). In addition, a low density of Forssman was observed over the ER and nuclear membrane, demonstrating that complex glycosphingolipids can be retrogradely transported to the ER (van Genderen et al. 1991). A number of glycolipid-binding toxins like cholera toxin (GM1) and Shiga toxin (Gb3) need to be transported back to the ER where their active subunit passes the membrane into the cytosol. It is not clear whether such transport reflects the natural retrograde transport of glycolipids or that it is induced by the pentameric toxins (Falguieres et al. 2006; Chinnapen et al. 2007), but GM1 has been found in the nuclear membrane by itself (Ledeen and $\mathrm{Wu} 2006$ ).

Although mitochondria generally contain very low levels of SM (van Meer 1989), a remarkable increase has been reported for cancer cells (see Holthuis et al. 2001) and mitochondrial SM appears to be the precursor for the apoptotic mitochondrial ceramide (Birbes et al. 2001). Likewise, by immuno-electron microscopy mitochondria were negative for Forssman labeling (van Genderen et al. 
1991) and for the disialo-ganglioside GD3 (Rippo et al. 2000; Garcia-Ruiz et al. 2002). However, mitochondrial GD3 labeling was found in Fas- and ceramide-induced apoptosis in lymphocytes (Rippo et al. 2000) and in TNF- $\alpha$ treated hepatocytes (Garcia-Ruiz et al. 2002). Unfortunately, these papers did not provide data on the GD3 labeling of the other cellular organelles leaving many unanswered questions concerning methodological controls and possible mechanisms of how GD3 got into the mitochondria. In any case, if the GD3 was originally synthesized in the lumen of the proximal Golgi (Uliana et al. 2006), it must have crossed a cellular membrane. This transmembrane translocation may have occurred as a consequence of the signaling at the plasma membrane (by the elusive scramblase?) or after retrograde traffic to the ER. Like all lipids tested so far GD3 may spontaneously translocate across the ER membrane. Subsequently, from the cytosolic surface of the ER it may reach the mitochondria, either via the ER-mitochondria contact sites or via monomeric transport through the cytosol, possibly stimulated by, e.g., the glycolipid transfer protein.

The fact that complex glycosphingolipids like Forssman glycolipid have been located to the ER and nuclear membrane and the indiscriminate flip-flop of lipids across the ER membrane suggests that SM and glycosphingolipids in general may occur to some extent in the cytosolic bilayer leaflet of the secretory and endocytotic organelles. Glucosylceramide is a special case in that it is synthesized on the cytosolic surface of the Golgi. Although many interactions between glycosphingolipids and cytosolic proteins have been reported over the years, a number of these studies were carried out in vitro and have not been validated in intact cells. In addition, when microscopic co-localization between glycosphingolipids and cytoskeletal elements was observed (i) often redistribution of the glycosphingolipid during sample preparation and labeling has not been thoroughly excluded, and (ii) the glycosphingolipids may have resided in the lumen of transport vesicles attached to the cytoskeleton (Gillard et al. 1993). Still, glycosphingolipids could in principle be present in complexes with proteins in the cytosol and nuclear matrix. A growing body of evidence supports the presence of intact phosphoinositides in the nuclear matrix, where they are thought to take part in signaling (Irvine 2006), and the same may be the case for SM and glycosphingolipids (Ledeen and $\mathrm{Wu} 2006$ ).

A special case are the sphingolipids ceramide, ceramide-1phosphate, sphingosine and sphingosine-1-phosphate which occur both as metabolic intermediates and as signaling lipids. It would be very important to be able to follow their local concentrations in time. However, there are several technical problems: (i) They are very sensitive to metabolic turnover (even during sample preparation), (ii) The single chain sphingosine and sphingosine-1-phosphate rapidly exchange as monomers through the aqueous phase, (iii) ceramide spontaneously flips across membranes, and finally (Rippo et al. 2000) there are only limited tools for their localization, like anti-ceramide antibodies (Vielhaber et al. 2001a) and it has not been established how well they label ceramide when it is present in different lipid and protein environments.

\section{Perspectives}

Glycosphingolipids and SM fulfill important functions within specialized domains of cellular membranes and in their interactions with proteins in, on and outside membranes. A combination of biological, physical, and chemical approaches is required to uncover these functions and the underlying molecular interactions. Morphology may be a great help. However, the traditional methodology is full of pitfalls. Notably, the specificity of the tools (Yanagisawa et al. 2006) and the fixation protocol (Schwarz and Futerman 1996; Heffer-Lauc et al. 2005) have been problematic. It is a tremendous challenge to try and overcome these methodological problems, and to be aware of them is a good start. All eukaryotes have sphingolipids and they display a bewildering range of structures. Evolution must have used their chemical and physical potential and endowed them with vital functions. Let's uncover them.

\section{Acknowledgments}

This work was supported by grant 805.47 .084 of the program From Molecule to Cell of the Netherlands Foundations for Earth and Life Sciences and Chemical Research (NWO-ALW and NWO-CW) with financial aid from the Netherlands Organization for Scientific Research. We are very grateful for a grant from the Mizutani Foundation for Glycoscience (G. van Meer).

\section{References}

Albi E. and Viola Magni M. P. (2004) The role of intranuclear lipids. Biol. Cell 96, 657-667.

Barbat C., Trucy M., Sorice M., Garofalo T., Manganelli V., Fischer A. and Mazerolles F. (2007) p56 lck, LFA-1 and PI3 K but not SHP-2 interact with GM1- or GM3-enriched microdomains in a CD4/p56 lck association-dependent manner. Biochem. J. 402, 471-481.

Barbosa M. L. F. and Pinto da Silva P. (1983) Restriction of glycolipids to the outer half of a plasma membrane: Concanavalin A labeling of membrane halves in acanthamoeba castellanii. Cell 33, 959-966.

Birbes H., El Bawab S., Hannun Y. A. and Obeid L. M. (2001) Selective hydrolysis of a mitochondrial pool of sphingomyelin induces apoptosis. FASEB J. 15, 2669-2679.

Bollinger C. R., Teichgraber V. and Gulbins E. (2005) Ceramide-enriched membrane domains. Biochim. Biophys. Acta 1746, 284-294.

Boot R. G., Verhoek M., Donker-Koopman W., Strijland A., van Marle J., Overkleeft H. S., Wennekes T. and Aerts J. M. (2007) Identification of the non-lysosomal glucosylceramidase as betaglucosidase 2. J. Biol. Chem. 282, 1305-1312.

Bosio A., Binczek E. and Stoffel W. (1996) Functional breakdown of the lipid bilayer of the myelin membrane in central and peripheral nervous system by disrupted galactocerebroside synthesis. Proc. Natl Acad. Sci. USA 93, 13280-13285. 
Brügger B., Graham C., Leibrecht I., Mombelli E., Jen A., Wieland F. and Morris R. (2004) The membrane domains occupied by glycosylphosphatidylinositol-anchored prion protein and Thy-1 differ in lipid composition. J. Biol. Chem. 279, 7530-7536.

Butor C., Stelzer E. H., Sonnenberg A. and Davoust J. (1991) Apical and basal Forssman antigen in MDCK II cells: a morphological and quantitative study. Eur. J. Cell Biol. 56, 269-285.

Chester M. A. (1998) IUPAC-IUB Joint Commission on Biochemical Nomenclature (JCBN). Nomenclature of glycolipids. Recommendations 1997. Eur. J. Biochem. 257, 293-298.

Chinnapen D. J., Chinnapen H., Saslowsky D. and Lencer W. I. (2007) Rafting with cholera toxin: endocytosis and trafficking from plasma membrane to ER. FEMS Microbiol. Lett. 266, 129-137.

Coetzee T., Fujita N., Dupree J., Shi R., Blight A., Suzuki K., Suzuki K. and Popko B. (1996) Myelination in the absence of galactocerebroside and sulfatide: normal structure with abnormal function and regional instability. Cell 86, 209-219.

Downes C. P., Gray A. and Lucocq J. M. (2005) Probing phosphoinositide functions in signaling and membrane trafficking. Trends Cell Biol. 15, 259-268.

Dragsten P. R., Blumenthal R. and Handler J. S. (1981) Membrane asymmetry in epithelia: is the tight junction a barrier to diffusion in the plasma membrane? Nature 294, 718-722.

Fahy E., Subramaniam S., Brown H. A. et al. (2005) A comprehensive classification system for lipids. J. Lipid Res. 46, 839-862.

Falguieres T., Romer W., Amessou M., Afonso C., Wolf C., Tabet J. C., Lamaze C. and Johannes L. (2006) Functionally different pools of Shiga toxin receptor, globotriaosyl ceramide, in HeLa cells. FEBS J. 273, 5205-5218.

Fra A. M., Masserini M., Palestini P., Sonnino S. and Simons K. (1995) A photo-reactive derivative of ganglioside GM1 specifically cross-links VIP21-caveolin on the cell surface. FEBS Lett. 375, $11-14$.

Fujimoto T. (1996) GPI-anchored proteins, glycosphingolipids, and sphingomyelin are sequestered to caveolae only after crosslinking. J. Histochem. Cytochem. 44, 929-941.

Futerman A. H. and van Meer G. (2004) The cell biology of lysosomal storage disorders. Nat. Rev. Mol. Cell Biol. 5, 554-565.

Garcia-Ruiz C., Colell A., Morales A., Calvo M., Enrich C. and Fernandez-Checa J. C. (2002) Trafficking of ganglioside GD3 to mitochondria by tumor necrosis factor-alpha. J. Biol. Chem. 277, 36443-36448.

Gillard B. K., Thurmon L. T. and Marcus D. M. (1993) Variable subcellular localization of glycosphingolipids. Glycobiology 3, 57-67.

Gomez-Mouton C., Abad J. L., Mira E., Lacalle R. A., Gallardo E., Jimenez-Baranda S., Illa I., Bernad A., Manes S. and Martinez A. C. (2001) Segregation of leading-edge and uropod components into specific lipid rafts during T cell polarization. Proc. Natl Acad. Sci. USA 98, 9642-9647.

Hakomori S. I. (2002) Inaugural article: the glycosynapse. Proc. Natl Acad. Sci. USA 99, 225-232.

Hanada K., Kumagai K., Yasuda S., Miura Y., Kawano M., Fukasawa M. and Nishijima M. (2003) Molecular machinery for non-vesicular trafficking of ceramide. Nature 426, 803-809.

Heffer-Lauc M., Lauc G., Nimrichter L., Fromholt S. E. and Schnaar R. L. (2005) Membrane redistribution of gangliosides and glycosylphosphatidylinositol-anchored proteins in brain tissue sections under conditions of lipid raft isolation. Biochim. Biophys. Acta 1686, 200-208.

Holowka D., Gosse J. A., Hammond A. T., Han X., Sengupta P., Smith N. L., Wagenknecht-Wiesner A., Wu M., Young R. M. and Baird B. (2005) Lipid segregation and IgE receptor signaling: a decade of progress. Biochim. Biophys. Acta 1746, 252-259.
Holthuis J. C., Pomorski T., Raggers R. J., Sprong H. and van Meer G. (2001) The organizing potential of sphingolipids in intracellular membrane transport. Physiol. Rev. 81, 1689-1723.

Hunt A. N. and Postle A. D. (2006) Mass spectrometry determination of endonuclear phospholipid composition and dynamics. Methods 39, 104-111.

Ichikawa S., Sakiyama H., Suzuki G., Hidari K. I. and Hirabayashi Y. (1996) Expression cloning of a cDNA for human ceramide glucosyltransferase that catalyzes the first glycosylation step of glycosphingolipid synthesis. Proc. Natl Acad. Sci. USA 93, 4638-4643.

Irvine R. F. (2006) Nuclear inositide signalling - expansion, structures and clarification. Biochim. Biophys. Acta 1761, 505-508.

Ishitsuka R., Sato S. B. and Kobayashi T. (2005) Imaging lipid rafts. J. Biochem. 137, 249-254.

Iwabuchi K., Handa K. and Hakomori S. (1998) Separation of 'glycosphingolipid signaling domain' from caveolin-containing membrane fraction in mouse melanoma B16 cells and its role in cell adhesion coupled with signaling. J. Biol. Chem. 273, 33766-33773.

James P. S., Hennessy C., Berge T. and Jones R. (2004) Compartmentalisation of the sperm plasma membrane: a FRAP, FLIP and SPFI analysis of putative diffusion barriers on the sperm head. $J$. Cell Sci. 117, 6485-6495.

Kirschning E., Rutter G. and Hohenberg H. (1998) High-pressure freezing and freeze-substitution of native rat brain: suitability for preservation and immunoelectron microscopic localization of myelin glycolipids. J. Neurosci. Res. 53, 465-474.

Kiyokawa E., Baba T., Otsuka N., Makino A., Ohno S. and Kobayashi T. (2005) Spatial and functional heterogeneity of sphingolipid-rich membrane domains. J. Biol. Chem. 280, 24072-24084.

Kolter T. and Sandhoff K. (2005) Principles of lysosomal membrane digestion: stimulation of sphingolipid degradation by sphingolipid activator proteins and anionic lysosomal lipids. Annu. Rev. Cell Dev. Biol. 21, 81-103.

Kuerschner L., Ejsing C. S., Ekroos K., Shevchenko A., Anderson K. I. and Thiele C. (2005) Polyene-lipids: a new tool to image lipids. Nat. Methods 2, 39-45.

Ledeen R. W. and Wu G. (2006) Sphingolipids of the nucleus and their role in nuclear signaling. Biochim. Biophys. Acta 1761, 588-598.

Lisanti M. P., Scherer P. E., Tang Z. and Sargiacomo M. (1994) Caveolae, caveolin and caveolin-rich membrane domains: a signalling hypothesis. Trends Cell Biol. 4, 231-235.

Madore N., Smith K. L., Graham C. H., Jen A., Brady K., Hall S. and Morris R. (1999) Functionally different GPI proteins are organized in different domains on the neuronal surface. $E M B O J . \mathbf{1 8}$, 6917-6926.

Manes S., Mira E., Gomez-Mouton C., Lacalle R. A., Keller P., Labrador J. P. and Martinez A. C. (1999) Membrane raft microdomains mediate front-rear polarity in migrating cells. EMBO J. 18, 6211-6220.

Maneta-Peyret L., Compere P., Moreau P., Goffinet G. and Cassagne C. (1999) Immunocytochemistry of lipids: chemical fixatives have dramatic effects on the preservation of tissue lipids. Histochem. J. 31, 541-547.

Mayor S., Viola A., Stan R. V. and del Pozo M. A. (2006) Flying kites on slippery slopes at Keystone. Symposium on lipid rafts and cell function. EMBO Rep 7, 1089-1093.

McGookey D. J., Fagerberg K. and Anderson R. G. (1983) Filipincholesterol complexes form in uncoated vesicle membrane derived from coated vesicles during receptor-mediated endocytosis of low density lipoprotein. J. Cell Biol. 96, 12731278. 
Mukherjee S. and Maxfield F. R. (2000) Role of membrane organization and membrane domains in endocytic lipid trafficking. Traffic 1, 203-211.

Negreiros E. M., Leao A. C., Santiago M. F. and Mendez-Otero R. (2003) Localization of ganglioside 9-O-acetyl GD3 in point contacts of neuronal growth cones. J. Neurobiol. 57, 31-37.

Pagano R. E. and Sleight R. G. (1985) Defining lipid transport pathways in animal cells. Science 229, 1051-1057.

Pagano R. E., Longmuir K. J., Martin O. C. and Struck D. K. (1981) Metabolism and intracellular localization of a fluorescently labeled intermediate in lipid biosynthesis within cultured fibroblasts. J. Cell Biol. 91, 872-877.

Pagano R. E., Martin O. C., Kang H. C. and Haugland R. P. (1991) A novel fluorescent ceramide analogue for studying membrane traffic in animal cells: accumulation at the Golgi apparatus results in altered spectral properties of the sphingolipid precursor. J. Cell Biol. 113, 1267-1279.

Parton R. G. (1994) Ultrastructural localization of gangliosides; GM1 is concentrated in caveolae. J. Histochem. Cytochem. 42, 155-166.

Pierce S. K. (2002) Lipid rafts and B-cell activation. Nat. Rev. Immunol. 2, 96-105.

Pike L. J. (2006) Rafts defined: a report on the keystone symposium on lipid rafts and cell function. J. Lipid Res. 47, 1597-1598.

Plowman S. J., Muncke C., Parton R. G. and Hancock J. F. (2005) H-ras, $\mathrm{K}$-ras, and inner plasma membrane raft proteins operate in nanoclusters with differential dependence on the actin cytoskeleton. Proc. Natl Acad. Sci. USA 102, 15500-15505.

Proia R. L. (2003) Glycosphingolipid functions: insights from engineered mouse models. Philos. Trans. Roy. Soc. Lond. B Biol. Sci. 358, 879-883.

Rintoul D. A. and Simoni R. D. (1977) Incorporation of a naturally occurring fluorescent fatty acid into lipids of cultured mammalian cells. J. Biol. Chem. 252, 7916-7918.

Rippo M. R., Malisan F., Ravagnan L. et al. (2000) GD3 ganglioside directly targets mitochondria in a bcl-2-controlled fashion. FASEB J. 14, 2047-2054.

Roux A., Cuvelier D., Nassoy P., Prost J., Bassereau P. and Goud B. (2005) Role of curvature and phase transition in lipid sorting and fission of membrane tubules. EMBO J. 24, 1537-1545.

Saito T. and Yokosuka T. (2006) Immunological synapse and microclusters: the site for recognition and activation of T cells. Curr. Opin. Immunol. 18, 305-313.

Schulte S. and Stoffel W. (1993) Ceramide UDPgalactosyltransferase from myelinating rat brain: purification, cloning, and expression. Proc. Natl Acad Sci. USA 90, 10265-10269.

Schwarz A. and Futerman A. H. (1996) The localization of gangliosides in neurons of the central nervous system: the use of anti-ganglioside antibodies. Biochim. Biophys. Acta 1286, 247.

Selvaraj V., Asano A., Buttke D. E. et al. (2006) Segregation of micronscale membrane sub-domains in live murine sperm. J. Cell. Physiol. 206, 636-646.

Sharma D. K., Choudhury A., Singh R. D., Wheatley C. L., Marks D. L. and Pagano R. E. (2003) Glycosphingolipids internalized via caveolar-related endocytosis rapidly merge with the clathrin pathway in early endosomes and form microdomains for recycling. J. Biol. Chem. 278, 7564-7572.

Sillence D. J., Raggers R. J. and van Meer G. (2000) Assays for transmembrane movement of sphingolipids. Methods Enzymol. 312, $562-579$

Simons K. and van Meer G. (1988) Lipid sorting in epithelial cells. Biochemistry 27, 6197-6202.

Simpson M. A., Cross H., Proukakis C. et al. (2004) Infantile-onset symptomatic epilepsy syndrome caused by a homozygous loss-offunction mutation of GM3 synthase. Nat. Genet. 36, 1225-1229.
Sklar L. A., Hudson B. S. and Simoni R. D. (1975) Conjugated polyene fatty acids as membrane probes: preliminary characterization. Proc. Natl Acad. Sci. USA 72, 1649-1653.

Somerharju P. (2002) Pyrene-labeled lipids as tools in membrane biophysics and cell biology. Chem. Phys. Lipids 116, 57-74.

Sorice M., Parolini I., Sansolini T., Garofalo T., Dolo V., Sargiacomo M., Tai T., Peschle C., Torrisi M. R. and Pavan A. (1997) Evidence for the existence of ganglioside-enriched plasma membrane domains in human peripheral lymphocytes. J. Lipid Res. 38, 969-980.

Spiegel S., Kassis S., Wilchek M. and Fishman P. H. (1984) Direct visualization of redistribution and capping of fluorescent gangliosides on lymphocytes. J. Cell Biol. 99, 1575-1581.

Sprong H., van der Sluijs P. and van Meer G. (2001) How proteins move lipids and lipids move proteins. Nat. Rev. Mol. Cell Biol. 2, 504-513.

Steer C. J., Bisher M., Blumenthal R. and Steven A. C. (1984) Detection of membrane cholesterol by filipin in isolated rat liver coated vesicles is dependent upon removal of the clathrin coat. J. Cell Biol. 99, 315-319.

Sugii S., Reid P. C., Ohgami N., Shimada Y., Maue R. A., Ninomiya H., Ohno-Iwashita Y. and Chang T. Y. (2003) Biotinylated theta-toxin derivative as a probe to examine intracellular cholesterol-rich domains in normal and Niemann-Pick type C1 cells. J. Lipid Res. 44, 1033-1041.

Svennerholm L. (1963) Chromatographic separation of human brain gangliosides. J. Neurochem. 10, 613-623.

Tillack T. W., Allietta M., Moran R. E. and Young Jr. W. W. (1983) Localization of globoside and forssman glycolipids on erythrocyte membranes. Biochim. Biophys. Acta 733, 15-24.

Uliana A. S., Giraudo C. G. and Maccioni H. J. (2006) Cytoplasmic tails of SialT2 and GalNAcT impose their respective proximal and distal Golgi localization. Traffic 7, 604-612.

Van Genderen I. L., van Meer G., Slot J. W., Geuze H. J. and Voorhout W. F. (1991) Subcellular localization of Forssman glycolipid in epithelial MDCK cells by immuno-electronmicroscopy after freeze-substitution. J. Cell Biol. 115, 1009-1019.

Van 't Hof W., Silvius J., Wieland F. and van Meer G. (1992) Epithelial sphingolipid sorting allows for extensive variation of the fatty acyl chain and the sphingosine backbone. Biochem. J. 283, 913-917.

Van Meer G. (1989) Lipid traffic in animal cells. Annu. Rev. Cell Biol. 5, 247-275.

Van Meer G. and Holthuis J. C. (2000) Sphingolipid transport in eukaryotic cells. Biochim. Biophys. Acta 1486, 145-170.

Van Meer G. and Simons K. (1986) The function of tight junctions in maintaining differences in lipid composition between the apical and the basolateral cell surface domains of MDCK cells. EMBO J. 5, 1455-1464.

Van Meer G., Stelzer E. H., Wijnaendts-van-Resandt R. W. and Simons K. (1987) Sorting of sphingolipids in epithelial (Madin-Darby canine kidney) cells. J. Cell Biol. 105, 1623-1635.

Vanier M. T. (1999) Lipid changes in Niemann-Pick disease type C brain: personal experience and review of the literature. Neurochem. Res. 24, 481-489.

Vielhaber G., Brade L., Lindner B., Pfeiffer S., Wepf R., Hintze U., Wittern K. P. and Brade H. (2001a) Mouse anti-ceramide antiserum: a specific tool for the detection of endogenous ceramide. Glycobiology 11, 451-457.

Vielhaber G., Pfeiffer S., Brade L., Lindner B., Goldmann T., Vollmer E., Hintze U., Wittern K. P. and Wepf R. (2001b) Localization of ceramide and glucosylceramide in human epidermis by immunogold electron microscopy. J. Invest. Dermatol. 117, 1126-1136.

Vyas K. A., Patel H. V., Vyas A. A. and Schnaar R. L. (2001) Segregation of gangliosides GM1 and GD3 on cell membranes, isolated membrane rafts, and defined supported lipid monolayers. Biol. Chem. 382, 241-250. 
Wang T. Y. and Silvius J. R. (2000) Different sphingolipids show differential partitioning into sphingolipid/cholesterol-rich domains in lipid bilayers. Biophys. J. 79, 1478-1489.

Wattenberg B. W. and Silbert D. F. (1983) Sterol partitioning among intracellular membranes. Testing a model for cellular sterol distribution. J. Biol. Chem. 258, 2284-2289.

Wilson B. S., Steinberg S. L., Liederman K., Pfeiffer J. R., Surviladze Z., Zhang J., Samelson L. E., Yang L. H., Kotula P. G. and Oliver J. M. (2004) Markers for detergent-resistant lipid rafts occupy distinct and dynamic domains in native membranes. Mol. Biol. Cell 15, 2580-2592.
Yamashita T., Wada R., Sasaki T., Deng C., Bierfreund U., Sandhoff K. and Proia R. L. (1999) A vital role for glycosphingolipid synthesis during development and differentiation. Proc. Natl Acad. Sci. USA 96, 9142-9147.

Yamashita T., Hashiramoto A., Haluzik M. et al. (2003) Enhanced insulin sensitivity in mice lacking ganglioside GM3. Proc. Natl Acad. Sci. USA 100, 3445-3449.

Yanagisawa M., Ariga T. and Yu R. K. (2006) Cholera toxin B subunit binding does not correlate with GM1 expression: a study using mouse embryonic neural precursor cells. Glycobiology 16, $19 \mathrm{G}-22 \mathrm{G}$ 